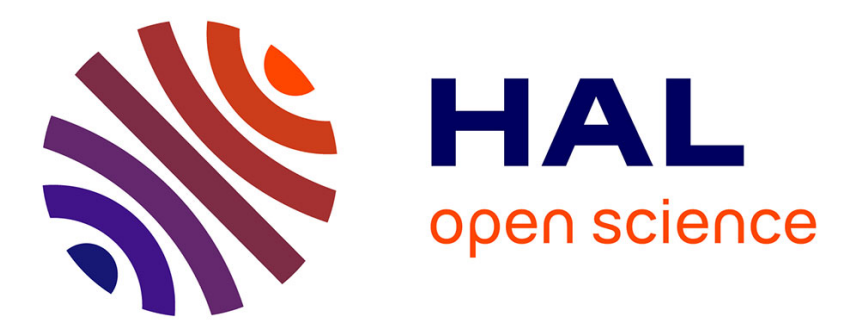

\title{
L'alimentation au Proche-Orient ancien : les sources et leur exploitation
}

\author{
Cécile Michel
}

\section{To cite this version:}

Cécile Michel. L'alimentation au Proche-Orient ancien: les sources et leur exploitation. L'histoire de l'alimentation dans l'Antiquité: Bilan historiographique, 2011, Paris, France. pp.17-45. halshs01187047

\section{HAL Id: halshs-01187047 https://shs.hal.science/halshs-01187047}

Submitted on 26 Aug 2015

HAL is a multi-disciplinary open access archive for the deposit and dissemination of scientific research documents, whether they are published or not. The documents may come from teaching and research institutions in France or abroad, or from public or private research centers.
L'archive ouverte pluridisciplinaire HAL, est destinée au dépôt et à la diffusion de documents scientifiques de niveau recherche, publiés ou non, émanant des établissements d'enseignement et de recherche français ou étrangers, des laboratoires publics ou privés. 
in B. Lion (éd.), L'histoire de l'alimentation dans l'Antiquité : Bilan historiographique, Dialogues d'Histoire Ancienne Supplément 7, Besançon, 2012, p. 17-45.

\section{L'alimentation au Proche-Orient ancien : les sources et leur exploitation}

Cécile Michel ${ }^{*}$

\section{Résumé}

Pour reconstituer l'alimentation des habitants du Proche-Orient ancien, les chercheurs disposent de sources archéologiques, iconographiques et textuelles. Ces dernières, abondantes, concernent en priorité la table des élites et les rations allouées aux travailleurs. Les vestiges archéologiques sont souvent des objets mis au rebut (tessons de vaisselle commune), des installations culinaires (meules, fours, silos), ainsi que des dépôts funéraires. Ces différents types de sources semblent, a priori, documenter des aspects différents de l'alimentation des anciens. Néanmoins, l'archéologie orientale a développé de nouvelles méthodes, depuis une vingtaine d'années, permettant de multiplier les informations sur les aliments: archéobotanique et archéozoologie, par exemple pour l'étude des restes végétaux et animaux. Il est donc possible de confronter les données archéologiques à celles fournies par les textes et les images. Trois exemples consacrés à l'huile, aux bols à forme tronconique et aux suidés sont présentés en détail.

Mots-clés

Alimentation, Proche-Orient ancien, huile, vaisselle, suidés.

\section{Abstract}

In order to recreate the diet of the Ancient Near Eastern people, researchers use archaeological remains, images and texts. These, quite numerous, refer first the table of the elites and the rations distributed to workers. Archaeological remains are often rubbish (fragments of common pots), culinary facilities (millstone, oven, silos) and funerary deposits. These various sources seem to deal with different aspects of food. But, Near Eastern archaeology developed these last decades, new methods providing new data on food: archaeobotanic and archaeozoology for example, for the study of plant and animal remains. It is now possible to cross archaeological data with texts and images. Three samples dedicated to oil, beveled rim bowls and pigs are analyzed.

Key words

Food, Ancient Near East, oil, vessel, pigs.

Pain et bière représentent nourriture et boisson par excellence des habitants du Proche-Orient ancien ; composés principalement d'orge, ils résultent du savoir-faire de l'être civilisé. Enkidu, le rival sauvage de Gilgamesh, roi d'Uruk, créé par les dieux pour mettre fin aux agissements tyranniques de ce dernier, vit nu, broute et boit l'eau claire des sources : «Enkidu ne savait pas comment manger du pain, comment boire de la bière, il n'avait pas encore appris » La prostituée qui accompagne Enkidu lui offre les explications suivantes : «Mange le pain, Enkidu, c'est ce qu'il faut pour vivre, bois la bière, c'est l'usage du pays » ${ }^{1}$. Il s'agit là d'actes vitaux et quotidiens. Or tout ce qui touche à la vie quotidienne échappe le plus souvent à l'écrit. Ces aspects sont mieux connus par les données archéologiques, qu'il s'agisse de la vaisselle ordinaire, des fours, silos, meules, restes végétaux et animaux.

Les archives privées livrent toutefois quelques informations sur d'occasionnels repas de fêtes qui célèbrent un mariage ou la conclusion d'un contrat : le partage de nourriture renforce les relations sociales ${ }^{2}$. Les banquets offerts par les souverains à leurs sujets sont décrits avec luxe de détails dans les sources officielles, véhicules de l'idéologie royaume. L'iconographie complète ces données par la représentation de scènes de banquet $^{3}$.

Cette répartition des sources, qui semblent, a priori, documenter des aspects différents de l'alimentation des anciens, explique, en partie, l'intérêt premier des assyriologues pour l'alimentation des élites, à commencer par les banquets royaux.

Depuis un quart de siècle, l'archéologie orientale a développé de nouvelles méthodes, permettant de multiplier les informations sur les aliments : archéobotanique et archéozoologie, par exemple pour l'étude des restes végétaux et animaux. Les épigraphistes, quant à eux, s'intéressent de plus en plus aux archives du

\footnotetext{
${ }^{*}$ ArScAn-HAROC, Maison René-Ginouvès, Archéologie et Ethnologie, Nanterre.

${ }^{1}$ Bottéro 1992a, 224 (passage extrait de la version paléo-babylonienne de Gilgameš).

${ }^{2}$ Bottéro 2002, 153-159.

${ }^{3}$ Lion \& Michel 2003.
} 
in B. Lion (éd.), L'histoire de l'alimentation dans l'Antiquité : Bilan historiographique, Dialogues d'Histoire Ancienne Supplément 7, Besançon, 2012, p. 17-45.

quotidien. Désormais, on confronte volontiers les données archéologiques à celles fournies par les textes et les images. Après un rapide inventaire des sources dont on dispose sur le sujet et l'exploitation qui en a été proposée dans l'histoire de l'assyriologie, trois exemples mettant en lumière la manière dont les sources peuvent être croisées seront développés ci-dessous ; deux d'entre eux restent actuellement l'objet de débats.

\section{Les sources textuelles et leur exploitation}

Les assyriologues doivent faire face à une masse considérable de textes, toujours en cours de déchiffrement. Ces textes sont de natures très variées : documents officiels, administratifs, archives privées, textes littéraires, listes lexicales, voire textes techniques, ils documentent des aspects très différents de l'alimentation. En outre, les informations qu'ils délivrent sont plus ou moins fiables, selon le public auquel ils s'adressent : ainsi le nombre de mets proposés lors d'un banquet royal peut souffrir d'une inflation importante en accord avec l'image que le roi veut donner de sa prodigalitét ${ }^{4}$.

\subsection{A la table des élites et des dieux : sources officielles, administratives et mythologiques}

Les sources textuelles relatives à la table des souverains et des dieux ont fait l'objet de nombreux travaux ${ }^{5}$. Peu prolixes pour le $\mathrm{III}^{\mathrm{e}}$ millénaire, où l'on relève principalement des dépenses de denrées alimentaires à l'occasion de fêtes religieuses ou des repas partagés par le roi avec ses militaires et ses hauts fonctionnaires ${ }^{6}$, les sources se diversifient au début du $\mathrm{II}^{\mathrm{e}}$ millénaire. Les archives royales du palais de Mari, ville située sur le Moyen-Euphrate et fouillée par les Français depuis les années 1930, fournissent de nombreuses lettres riches en détails sur la recherche de bons cuisiniers, de mets rares ou exotiques (comme les truffes), ainsi que sur le déroulement des repas et l'accueil des ambassadeurs étrangers : les plus importants avaient droit à un siège, les autres étaient assis par terre ${ }^{7}$. D'autres renseignent sur les méthodes de conservation des denrées, ou encore sur leur transport ${ }^{8}$.

Les grandes institutions de la seconde moitié du $\mathrm{III}^{\mathrm{e}}$ millénaire et de la première moitié du $\mathrm{II}^{\mathrm{e}}$ millénaire, comme les palais de Mari ou d'autres villes de haute Mésopotamie (Chagar Bazar, Tell Leilan etc.), ont livré des milliers de documents administratifs. Ceux-ci témoignent de la gestion des biens du souverain, d'une part dans son royaume, comme les gigantesques parcs à bestiaux de Girsu, Puzriš-Dagan et Umma appartenant aux souverains de l'empire d'Ur III $^{9}$, et d'autre part à l'intérieur du palais, avec des bureaux spécialisés pour la viande, le vin, la bière ${ }^{10}$. À Mari, les archéologues ont mis au jour des centaines de billets quotidiens enregistrant les denrées sorties des magasins pour le repas quotidien du roi et de la cour ${ }^{11}$, et de nombreux textes donnant l'inventaire de la vaisselle précieuse accompagnant régulièrement le roi lors de ses voyages ${ }^{12}$. Les textes mythologiques montrent les dieux réunis autour de festins où la bière (en Mésopotamie) et le vin (à Ougarit) coulent à flots et où l'ivresse bat son plein ${ }^{13}$. Dans les temples, les dieux, par l'intermédiaire de leurs statues, consomment les offrandes régulières qui leur sont présentées ${ }^{14}$. Ainsi, les archives administratives des temples néo-babyloniens (seconde moitié du $\mathrm{I}^{\mathrm{er}}$ millénaire) détaillent les prébendes du personnel du clergé chargé de l'entretien des divinités.

Au $\mathrm{I}^{\mathrm{er}}$ millénaire, et plus particulièrement à la cour néo-assyrienne ( $\mathrm{IX}^{\mathrm{e}}-\mathrm{VII}{ }^{\mathrm{e}}$ siècles av. J.-C.), les banquets royaux prennent de l'envergure : le nombre de convives augmente, de même que la quantité et la variété des denrées consommées ; les convives boivent et mangent dans de la vaisselle de luxe. Les inscriptions royales détaillent ces moments festifs organisés par le souverain à l'occasion d'une victoire ou pour célébrer de grands travaux de construction (temple, palais, ville); les documents administratifs précisent la nature et la quantité des denrées. Grâce à la stèle d'Aššurnașirpal II, maintes fois commentée, on connaît avec précision le menu offert par le roi pour l'inauguration de sa nouvelle capitale, Kalhu, à ses 69574 convives pendant 10 jours $^{15}$. Des données similaires ont été analysées pour les sources perses ${ }^{16}$.

\footnotetext{
${ }^{4}$ Dans la plupart des cas, il s'agit de nombre ronds. C'est ainsi que sont dénombrées les denrées offertes par Aššurnazirpal II au banquet d'inauguration de sa nouvelle capitale, Kalhu; en revanche, le nombre précis de convives 69 574, dont 47074 personnes venant des différents districts du pays serait symbolique, cf. De Odorico 1995, 141-142.

${ }^{5}$ Gyselen 1992 ; Joannès 1996; Joannès 2008; Joannès 2009c ; Durand \& Jacquet s. p. ; Hugoniot, Grandjean \& Lion s. p.

${ }^{6}$ Steinkeller 2008.

${ }^{7}$ Charpin 1988, 139-205 ; Durand 1997, 333-372 et 383-614 ; Lafont 2001, 298-301.

${ }^{8}$ Charlier 1987 ; Joannès 1994 ; Michel 2009.

${ }^{9}$ Sigrist 1992 ; Brunke 2011 ; Lafont 2009.

${ }^{10}$ Durand 1981 ; Chambon 2009 ; Lacambre 2009.

${ }^{11}$ Bottéro 1957 ; Birot 1960 ; Birot 1964 ; Burke 1968 ; Durand 1983, 166-180 ; Lafont 1985 ; Sasson 2004.

${ }^{12}$ Guichard 2005.

${ }^{13}$ Michalowski 1994.

${ }^{14}$ Ces aspects ont été analysés à partir des archives des temples de la seconde moitié du Ier millénaire av. J.-C. : Joannès 2008 ; Parpola 2004.

${ }^{15}$ Finet 1992 ; Lion \& Michel 2003, 24-31.

${ }^{16}$ Briant 1989 ; Amigues 2003 ; Tolini 2009a ; Tolini 2009b ; Hugoniot, Grandjean \& Lion s. p.
} 
in B. Lion (éd.), L'histoire de l'alimentation dans l'Antiquité : Bilan historiographique, Dialogues d'Histoire Ancienne Supplément 7, Besançon, 2012, p. 17-45.

\subsection{Rations des travailleurs : sources administratives}

À partir du $\mathrm{III}^{\mathrm{e}}$ millénaire avant notre ère, les grands domaines agricoles appartenant aux palais et aux temples dominent l'économie de la Basse Mésopotamie ; une grande partie de la population dépend, pour sa subsistance, de son employeur, le plus souvent institutionnel. Les sources administratives témoignent de la rémunération des travailleurs par un système de rations, distribuées régulièrement et comportant orge, huile et laine; les quantités varient en fonction du sexe, de l'âge ou de la fonction exercée par le récipiendaire. Ce système de distribution de rations, qui perdure jusqu'à la fin de la civilisation mésopotamienne, a généré beaucoup de documents cunéiformes : listes de personnels classés par profession, régulièrement mises à jour, et notifications du versement des rations ${ }^{17}$. Il a suscité de nombreuses études, en particulier sur le régime alimentaire des employés, en qualité et quantité (cf. ci-dessous § 4.2).

\subsection{Les aliments d'après les listes lexicales}

Aujourd'hui, on classerait schématiquement en trois grandes catégories les aliments dont disposent les anciens Mésopotamiens : les aliments issus des végétaux (céréales, légumes, fruits, etc.), ceux provenant de la faune (principalement viande, graisse et lait) et les produits dérivés, résultant d'une transformation (pain, huile, bière, vin, etc.). Les habitants du Proche-Orient ancien procèdent également à une classification des réalités de la nature et de la société dans le cadre de l'apprentissage de la langue et de l'écriture. Au III ${ }^{\mathrm{e}}$ millénaire, avec le développement de cette écriture, les listes lexicales, en sumérien, se multiplient. Au milieu du $\mathrm{II}^{\mathrm{e}}$ millénaire, elles deviennent bilingues, en ajoutant à côté des mots sumériens leur traduction akkadienne, ce qui en font de véritables dictionnaires. La plus complète, $\mathrm{UR}_{5} \cdot \mathrm{RA}=$ hubullu, nommée d'après son incipit « la dette ${ }^{18}$, procède à un classement du vocabulaire sur 24 tablettes, dont un tiers se rapporte peu ou prou à l'alimentation :

- $\quad$ trois tablettes sont consacrées aux animaux (XIII : domestiques, XIV : sauvages, XVIII : poissons et oiseaux),

- $\quad$ la tablette XVII liste les plantes (pas toutes comestibles),

- $\quad$ la tablette XXII, les aliments liquides et les farines,

- la tablette XXIV rassemble d'autres aliments : miel, graisse, produits lactés, fruits et légumes et... le bitume. Le raisin ne figure pas dans cette dernière liste, mais la vigne est classée avec les arbres dans la tablette III.

À ces inventaires, on peut ajouter la tablette X qui donne les noms des fours, récipients et objets en argile, voire la tablette XI qui concerne les peaux, minéraux, cuivre et objets en cuivre, et donc la vaisselle.

La tablette XIII, consacrée aux animaux domestiques, réunit sur 400 lignes les animaux de rente, et plus particulièrement les ovins, caprins et bovins ${ }^{19}$. Les ovins, désignés par le signe du mouton, UDU, remplissent à eux seuls la moitié de la liste ; les animaux y sont classés selon leur sexe, leur âge, leur statut reproducteur et leur variété (morphologie, origine, couleur). La désignation des animaux, rangés selon des classificateurs spécifiques, obéit à différents critères qui tiennent compte à la fois de caractéristiques biologiques et des usages qu'en fait l'homme. Les suidés tiennent une place à part dans ces listes et feront l'objet d'un développement particulier (cidessous $§ 4.3)$.

La tablette XXIII, qui n'a pas pu être reconstituée dans sa totalité, énumère successivement les soupes, un ingrédient sec qui intervient dans la préparation de la bière (KAŠ DI.DA), la « bière » et ses variétés ou plus généralement les boissons fermentées alcoolisées (KAŠ), les «pains de bière », les différents types de moût, la purée chauffée de malt et céréales concassées, le malt ${ }^{20} \ldots$. Cette tablette fait donc la part belle à la bière et à ses ingrédients, et elle a permis de reconstituer les techniques de brasserie mésopotamiennes ${ }^{21}$.

\subsection{Quelques recettes et procédés de conservation}

Ces listes lexicales ont été l'une des sources d'inspiration de Jean Bottéro pour ses nombreux travaux sur l'alimentation au Proche-Orient ancien ${ }^{22}$, et plus particulièrement lorsqu'il a magistralement publié trois textes difficiles, datant $\mathrm{du} \mathrm{XVIII}^{\mathrm{e}}$ siècle avant notre ère et comportant des séries de recettes. Il s'agit principalement de préparations de bouillons avec ou sans viande ainsi que des pâtes et des tourtes. Les quantités des ingrédients, aux noms parfois inconnus, ne sont jamais indiquées, ni les temps de cuisson. En voici un

\footnotetext{
${ }^{17}$ Gelb 1965 ; Steinkeller 1987; Waetzoldt 1987; Maekawa 1989; Englund 1991 ; Steinkeller 1996; Maekawa 1998; Koslova 2006; Beaugeard 2009; Faivre 2009c ; Tolini 2009c

${ }^{18}$ Cavigneaux 1983 ; ces listes ont été publiées dans la collection initiée par B. Landsberger et intitulée Materials for the Sumerian lexicon (MSL), 5, 6, 7, 9, 10 and 11, Rome: Pontificium Institutum Biblicum, 1957 -

${ }^{19}$ Landsberger 1960. Voir également BSA 7 et 8.

${ }^{20}$ Reiner \& Civil 1974, 67-76.

${ }^{21}$ Hartman \& Oppenheim 1950 ; Röllig 1970; Stol 1971 ; Milano 1994 ; Breniquet 2009a ; 2009b ; Faivre 2009d ; Michel 2009c ; Michel 2009d.

${ }^{22}$ Bottéro 1995 ; Bottéro 2002. Pour un inventaire des nombreuses publications de J. Bottéro touchant à l'alimentation, cf. Faivre, Lion \& Michel 2009, 309-341.
} 
in B. Lion (éd.), L'histoire de l'alimentation dans l'Antiquité : Bilan historiographique, Dialogues d'Histoire Ancienne Supplément 7, Besançon, 2012, p. 17-45.

extrait, qui donne la recette de la tourte aux petits oiseaux ${ }^{23}:$ « Pour préparer une [...] de 'petits oiseaux', tu en détache la tête, le cou et les pattes; tu leur ouvres le ventre, et tu en retires gésier et fressure. Après avoir fendu le gésier, tu le pèles. Tu laves alors les oiseaux et tu (en) haches la fressure. Puis, ayant récuré un chaudron, tu y mets oiseaux, gésiers et intestins (oiseaux et abats sont cuits dans un bouillon avec des alliacées ». (Suivent des instructions pour faire une pâte à base de semoule et lait ; la pâte est divisée en deux, l'une est foncée dans un plat pour le fond de la tourte, l'autre préparée pour en faire le couvercle). « Juste avant le repas, tu prends le platprésentoir garni d'une abaisse, et tu y ranges avec soin les oiseaux cuits; par-dessus, tu parsèmes la fressure et les gésiers découpés, qui se trouvaient dans la marmite, ainsi que les pains qui avaient cuit au four (...) Tu recouvres de son 'couvercle' (en pâte) le plat-présentoir et tu le sers à table ${ }^{24}$.

Sans l'aide de textes techniques similaires, on a pu aussi reconstituer des recettes de pâtisserie grâce à des billets administratifs. Il s'agit de cakes ou pains d'épice de composition plus complexe, tel le mersu dont la recette consiste à mélanger de la farine avec de l'eau, du lait, de la bière, ou plus souvent huile ou beurre; cette pâte est fourrée de dattes, de raisin, de figues, de pommes ou de pistaches, et aromatisée avec du cumin, de la coriandre et de l'ail ${ }^{25}$. Du miel lui est ajouté pour le sucrer. Le tout est cuit au four, peut-être dans des moules ressemblant à ceux qui ont été découverts dans les cuisines du palais de Mari ${ }^{26}$. De même, listes lexicales, textes administratifs et archives privées ont permis de reconstituer les procédés de fabrication de la bière à partir de la macération ou fermentation d'orge. Il en existe de nombreuses variétés : bière noire, bière claire, bière rouge d'épeautre, bière de première qualité, bière triple, bière clarifiée ${ }^{27}$.

Avant d'être cuisinées, les denrées alimentaires sont parfois traitées pour être conservées, lorsque cela est possible. Ainsi, le lait qui ne se conserve pas, n'est que rarement mentionné dans les textes ${ }^{28}$. Archives administratives et privées mentionnent le séchage des céréales, la fumaison ou la salaison de la viande et du poisson, voire la fabrication de conserves de ragout pour les voyages ${ }^{29}$. Les procédés ne s'avèrent pas toujours efficaces et on recense trouve de la viande avariée, du vin moisi ou encore de la farine infestée de charançons.

\subsection{Archives privées}

Toutes ces techniques sont bien connues des particuliers comme en témoignent leurs archives et plus particulièrement leur correspondance, qui, dans certaines occasions, documentent divers aspects de l'alimentation quotidienne. C'est, par exemple, le cas des nombreuses archives des marchands assyriens, établis loin de chez eux, en Anatolie, et datant principalement du XIX ${ }^{\mathrm{e}}$ siècle av. J.-C. Les lettres envoyées par leurs épouses demeurées seules à Aššur, sur le Tigre, non loin de Mossul, où celles adressées à des femmes de Kaniš, en Anatolie centrale, à proximité de Kayseri, permettent de reconstituer leurs habitudes alimentaires, depuis l'acquisition des denrées, leur stockage, leur transformation, jusqu'à leur consommation ${ }^{30}$.

Les céréales ${ }^{31}$, surtout l'orge, mais aussi blé et épeautre, constituent la base de l'alimentation pour les hommes et un complément pour les animaux domestiques. Elles servent à la confection de farines, de gruaux, de pains et aussi de bière. Le pain, aliment par excellence, varie selon la farine utilisée, le liquide employé (huile, lait, bière), la présence ou l'absence de levain, la nature des épices qui le parfument ou des fruits qui le fourrent, la taille, l'épaisseur ou la forme! On moud avec une meule roulante les graines de céréales, orge, épeautre ou froment, pour obtenir de la farine et le pain est ensuite cuit à la maison. L'huile utilisée pour la cuisine est extraite du sésame (cf. ci-dessous $§ 4.1$ ), mais on utilise aussi les graisses animales.

Les légumes, surtout les féculents, sont employés en purées ou grillés, et complétés par d'autres légumes, principalement des alliacées. Le sel, les épices et les herbes aromatiques relèvent la saveur des aliments. Les fruits qui poussent dans les vergers sont assez variés: dattes, figues, pommes, poires, grenades, raisin, noix, noisettes, amandes et térébinthes.

La consommation de la viande est d'usage répandu, mais elle semble plus rare chez les gens de statut inférieur qui, selon les régions, se contentent parfois de poisson. Les ovins constituent la base de l'alimentation carnée $^{32}$. La boisson de base des anciens Mésopotamiens est la bière d'orge, fabriquée localement. Plus rare, le vin est importé de l'Ouest, conservé et sucré par le miel. L'eau n'apparaît jamais dans les textes, non que les

\footnotetext{
${ }^{23}$ Bottéro 1995, 114.

${ }^{24}$ Ces tourtes rappellent les nids d'oiseaux que les serviteurs d'Aššurbanipal rapportent de la chasse pour la table du roi, relief reproduit dans Matthiae 1998, 142-143.

${ }^{25}$ Sasson 2004.

${ }^{26}$ Parrot 1959, 33-57, pl. 12-37 ; Margueron 2004, 515-516.

${ }^{27}$ Cf. ci-dessus note 21 .

${ }^{28}$ Gouin 1993.

${ }^{29}$ Bottéro 1992 ; Guichard 1997 ; Lion \& Michel 1997 ; Lion \& Michel 2000 ; Sasson 2004 ; Chambon 2009 ; Patrier 2009a ; Michel 2009b ; van der Stede 2010 ; Marro \& Michel s. p.

${ }^{30}$ Michel 1997.

${ }^{31}$ Joannès 2001 : ce dictionnaire comporte de nombreuses entrées sur les aliments ou en lien avec l'alimentation.

${ }^{32}$ Michel 1997; Vila 1998; Michel 2006; Dercksen 2008.
} 
in B. Lion (éd.), L'histoire de l'alimentation dans l'Antiquité : Bilan historiographique, Dialogues d'Histoire Ancienne Supplément 7, Besançon, 2012, p. 17-45.

gens n'y goûtent pas, mais la présence de cruches d'eau à table va de soi ; en voyage l'eau est transportée dans des outres.

Ces denrées sont consommées au cours de deux repas quotidiens ${ }^{33}$. Le repas du matin, relativement tôt, comprend surtout du gruau, tandis que le repas du soir constitue le repas principal. Les plus pauvres s'asseyent autour d'un grand plat profond dans lequel chacun se sert à la main ${ }^{34}$, mais dans les textes sont mentionnés cuillers et couteaux, plateaux qui devaient reposer sur des supports. Les dots des femmes comportent des meules et de la vaisselle en métal. La céramique est rarement mentionnée dans les textes de la pratique, mais bien représentée dans l'iconographie et abondamment découverte sur les sites archéologiques.

\section{La représentation du banquet : sources iconographiques}

L'iconographie mésopotamienne est moins prolixe et détaillée que l'iconographie égyptienne. Les images appartiennent majoritairement aux domaines religieux et royal. Les scènes de la vie quotidienne y sont rares, excepté les représentations de banquets des élites. Au III ${ }^{\mathrm{e}}$ millénaire avant J.-C. apparaissent des scènes figurant plusieurs convives avec gobelet à la main, sur des sceaux-cylindres (comme celui de la reine d'Ur Puabi), des plaques en relief (la plaque d'Ur-Nanše, où le souverain de Lagaš fête avec sa famille l'achèvement de la construction d'un temple dédié aux dieux Ningirsu et Nanše) ou des mosaïques, dont la plus fameuse, l'Étendard d'Ur, présente un banquet célébrant une victoire sur la face dite de «la paix $»^{35}$.

$\mathrm{Au} \mathrm{I} \mathrm{I}^{\mathrm{er}}$ millénaire, les banquets néo-assyriens figurés sur les bas-reliefs qui ornent les murs des palais royaux font écho aux inscriptions royales contemporaines ${ }^{36}$. Sur les murs du palais de Sargon II à Khorsabad, les convives, des hommes, sont assis par deux ou par quatre sur des tabourets ornés. Le roi siège sur un trône à haut dossier, à l'écart des invités; il est entouré de plusieurs serviteurs chargés de le servir et de l'éventer. Les plats sont apportés par des eunuques et posés sur des plateaux disposés sur des tréteaux. Les invités boivent dans des coupes ornées de têtes de lion. Dans le palais de Sennacherib à Ninive, la scène commence en amont, avec des serviteurs qui acheminent les animaux et les produits divers qui vont alimenter le festin. Le Banquet sous la treille d'Aššurbanipal, représenté dans le palais nord de Ninive, est tout à fait atypique. Le roi et la reine festoient seuls, dans le parc du palais, après une victoire remportée sur l'Elam. Le roi mange, allongé sur un lit, la reine, à ses pieds, est assise sur un siège: il s'agit de la première attestation d'un repas couché ${ }^{37}$. Des musiciens accompagnent le repas et la tête de l'ennemi vaincu est accrochée à un arbre.

En marge de ces images sur le banquet, des plaques d'argile datant du début du $\mathrm{II}^{\mathrm{e}}$ millénaire montrent des personnages en train de boire de la bière à l'aide d'un chalumeau filtrant ${ }^{38}$. Ces scènes feraient échos à celles représentées en miniature sur les sceaux-cylindres dès la première moitié du IV millénaire (époques d'Uruk et Dynastique archaïque 3700-2330). L'interprétation de ces dernières, pour la période protodynastique, a été récemment remise en question par $\mathrm{C}$. Breniquet ${ }^{39}$ : elle a constaté en effet que les tiges qui émergent du récipient sont nombreuses et ne se dirigent pas vers la bouche des individus. Le motif de la boisson au chalumeau n'apparaîtrait en fait qu'à la période d'Akkad, au milieu du $\mathrm{III}^{\mathrm{e}}$ millénaire, et la majorité des scènes antérieures relèveraient de l'étirage de la laine : les protagonistes sont des femmes, assises, et les autres tiges sortant du vase pourraient être des quenouilles fichées là en attendant d'être utilisées. On voit là que les images peuvent poser d'importants problèmes d'identification.

Le domaine des cuisiniers est exceptionnellement représenté, comme par exemple sur un relief du palais d'Assurnazirpal II (883-859) qui montre la préparation du repas dans un camp circulaire ${ }^{40}$.

\section{Les données archéologiques : nourriture des vivants et des morts}

Les données archéologiques sur l'alimentation sont variées et pas toujours exploitées sur les sites ${ }^{41}$. Les archéologues du Proche-Orient ancien travaillent sur des tells de très grande taille, ce qui nécessite d'importants moyens humains. La plupart des sites importants, à commencer par Ninive, Kalhu et Dūr-Šarrukēn ont été fouillés dès le milieu du XIX $x^{\mathrm{e}}$, et surtout au début du XX $\mathrm{X}^{\mathrm{e}}$ siècle (Aššur et Babylone). Les archéologues de l'époque ont mis leurs efforts sur l'architecture, la statuaire et les tablettes couvertes de signes cunéiformes. Les objets de plus petite taille, et plus spécifiquement, ceux du quotidien, ont fait l'objet de moins d'attention.

Toutes ces données archéologiques illustrent bien entendu les habitudes alimentaires des habitants du Proche-Orient ancien, mais aussi de leurs dieux, par le biais des offrandes alimentaires, et des morts. En effet, se

\footnotetext{
${ }^{33}$ Glassner 1987/1990.

${ }^{34}$ Un relief du $\mathrm{VII}^{\mathrm{e}}$ siècle provenant du palais d'Aššurbanipal à Ninive montre des déportés élamites assis autour d'un récipient et mangeant avec leurs doigts ; ce relief est reproduit dans Bordreuil, Briquel-Chatonnet \& Michel 2008, p. 211.

${ }^{35}$ Selz 1983 ; Collon 1992 ; Schmandt-Besserat 2001 ; Pollock 2003.

${ }^{36}$ Barnett 1985 ; Pinnock 1994 ; Bachelot 2009a.

${ }^{37}$ Dentzer 1982.

${ }^{38}$ Voir par exemple la plaque de Harradum reproduite par Breniquet 2009a, p. 196, figure 11.

${ }^{39}$ Breniquet 2009b.

${ }^{40}$ Reproduit dans Lion \& Michel 2003, p. 55. Il existe également quelques reliefs présentant des déportés faisant un feu.

${ }^{41}$ Calmeyer 1987/1990.
} 
in B. Lion (éd.), L'histoire de l'alimentation dans l'Antiquité : Bilan historiographique, Dialogues d'Histoire Ancienne Supplément 7, Besançon, 2012, p. 17-45.

nourrir est une nécessité vitale, mais en Mésopotamie cette nécessité s'étend au-delà d'une vie humaine : les morts sont supposés mener eux aussi une forme de vie dans l'Au-delà ; ils doivent s'alimenter ou plutôt être alimentés par les vivants qui partagent occasionnellement un repas avec eux, dans leur espace ${ }^{42}$. Dans les tombes, de nombreux restes de repas ont ainsi était découverts et ont fait l'objet d'études détaillées, tout particulièrement en ce qui concerne les tombes royales de Qatna, en Syrie, datant du milieu du IIe millénaire et découvertes ces dernières années. Ces sources montrent que la nourriture était fournie aux défunts en diverses circonstances et donnent quelque éclairage sur la nature, les quantités et le rythme des offrandes alimentaires ${ }^{43}$.

\subsection{Les vestiges architecturaux : structures de stockage et cuisines}

Parmi les vestiges architecturaux, les structures de stockage, principalement des céréales, n'ont reçu l'attention des spécialistes que récemment ${ }^{44}:$ magasins à vivres, greniers, silos, celliers, etc. Les silos ont permis aux chercheurs d'effectuer quelques spéculations, mais il faut être très prudent : à Boğazköy, capitale du pays hittite, les silos du mur des poternes auraient pu contenir assez de grain pour nourrir 20000 à 35000 personnes chaque année ${ }^{45}$. Ces calculs ont-ils été effectués sur la base d'une alimentation uniquement céréalière ? A-t-on affaire avec un stockage communal ou étatique ? Qu'en est-il des réserves pour les semences ?

Pour rester en Anatolie, dans un quartier marchand habité par des Assyriens au début du II ${ }^{\mathrm{e}}$ millénaire, à Kültepe, on s'est rendu compte que chaque maison privée comportait un four parfois être de taille importante, pour cuire les aliments ${ }^{46}$ : cela implique-t-il un grand nombre d'habitants par maison ?

\subsection{Vestiges mobiliers}

Les vestiges mobiliers en rapport avec l'alimentation sont extrêmement variés, et il n'est pas question ici d'en faire un inventaire : les palais, temples, maisons privées et tombes ont livré de très nombreux contenants de toute taille, principalement en argile, plus rarement en métal ${ }^{47}$; bois et roseaux n'ont pas survécu au climat de la Mésopotamie. Dans les cuisines, on a également découvert meules et mortiers, et divers autres ustensiles témoignant d'opérations culinaires ${ }^{48}$.

Certaines maisons privées, par exemple dans le quartier marchand de Kültepe, recelaient une quantité impressionnante de céramiques. Comprendre l'usage qui était fait de ces récipients n'est pas toujours évident, mais l'étude des changements de forme permet parfois d'isoler des variations fonctionnelles ${ }^{49}$. Quelques chercheurs tentent de faire correspondre le vocabulaire attesté par les textes aux vases découverts sur le terrain.

\subsection{Alimentation carnée : archéozoologie}

L'archéozoologie s'est développée depuis un quart de siècle sur les sites du Proche-Orient ancien, avec un ramassage plus minutieux et systématique des restes fauniques ${ }^{50}$. On sait désormais quelles espèces animales étaient consommées et les traces analysées sur les os donnent des indices sur les procédés de découpe des animaux et leur préparation, deux étapes où les aspects techniques et culturels priment ${ }^{51}$. Ces analyses sont parfois complétées par des études ethnoarchéologiques.

\subsection{Céréales, fruits et légumes : archéobotanique}

On constate une évolution similaire pour les études archéobotaniques au Proche-Orient. Celles-ci se sont développées d'abord pour les époques pré- et protohistoriques, afin de caractériser les modes d'alimentation des populations de chasseurs-cueilleurs, puis des premiers agriculteurs et éleveurs ${ }^{52}$. Depuis les années 1980 , ces analyses ont été appliquées également pour les sites des périodes historiques ( $\mathrm{III}^{\mathrm{e}}-\mathrm{I}^{\mathrm{er}}$ millénaires), permettant désormais de confronter les résultats obtenus aux données textuelles (cf. ci-dessous $§ 4.1$ ). On étudie les fruits et graines (carpologie), les charbons de bois (anthracologie) et même les spores et graines de pollen fossiles (palynologie). Le Bulletin on Sumerian Agriculture (BSA), issu des réunions d'un groupe créé à cette époque, comporte plusieurs études archéobotaniques.

À titre d'exemple, l'étude des restes paléobotaniques pour la phœniciculture (fruits, graines, empreintes de nattes ou fragments de stipe), dans les sites du Proche-Orient datés de l'Âge du Bronze, a permis de

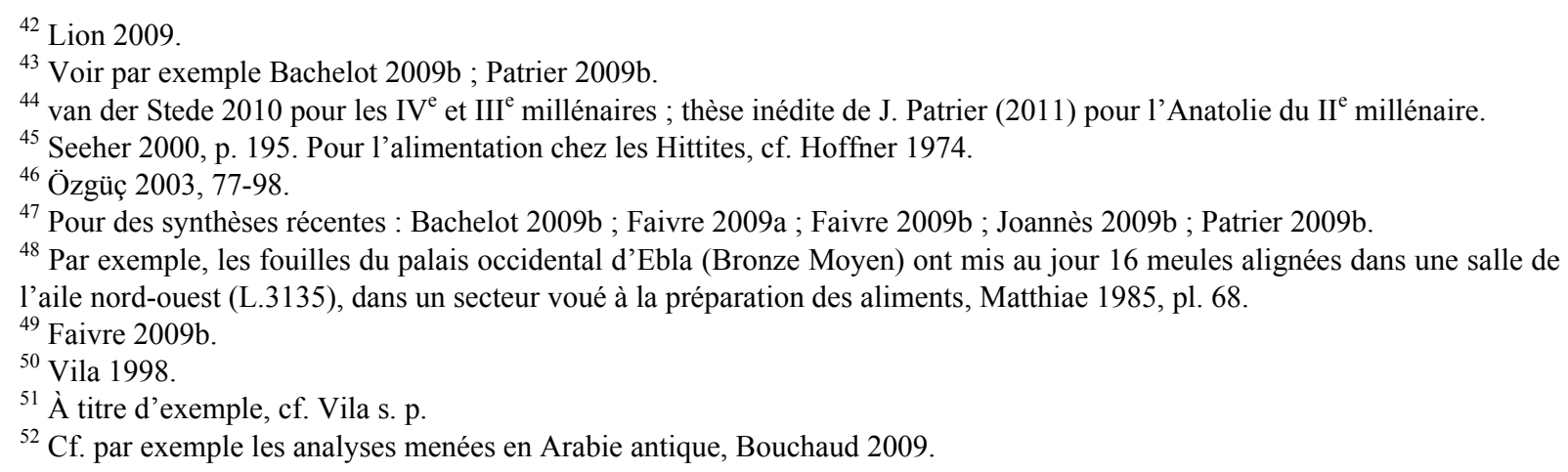


in B. Lion (éd.), L'histoire de l'alimentation dans l'Antiquité : Bilan historiographique, Dialogues d'Histoire Ancienne Supplément 7, Besançon, 2012, p. 17-45.

reconstituer la culture et les utilisations du palmier dattier ${ }^{53}$. Les aspects économiques de cette culture dans de grandes palmeraies d'époque néo-babylonienne appartenant aux palais et aux temples sont abondamment documentés par les textes. Les archives de l'Eanna, le temple d'Ištar à Uruk, illustrent la gestion sous forme de Ferme générale de toutes les palmeraies lui appartenant : l'ensemble fournissait une récolte annuelle de 18000 hectolitres ${ }^{54}$.

\subsection{Les analyses chimiques : indices pour des recettes ?}

D'autres traces repérées par l'archéologue apportent des indices sur le régime alimentaire comme les caries dentaires ou des analyses isotopiques des restes humains. On détecte ainsi diverses carences alimentaires 55 .

De même, les traces conservées dans certaines céramiques qui n'ont pas subi un nettoyage trop énergique permettent parfois d'identifier les produits qui y étaient stockés. Les analyses menées sur les résidus présents dans de grands récipients à fond percé du site de Tell Bazi (en Syrie) ont révélé la présence d'oxalate de calcium et de levures microscopiques permettant la germination pour le maltage de l'orge. Un important travail de reconstitution du brassage de la bière dans l'antiquité a été mené en collaboration avec des spécialistes de la brasserie contemporaine ${ }^{56}$; au dire des archéologues, les résultats expérimentaux obtenus n'étaient pas si mauvais.

\section{Croiser les sources}

Le croisement des différentes sources fournies par l'archéologie, les images et les textes a permis des progrès importants dans l'identification de vestiges mobiliers liés à l'alimentation, la traduction de certains termes ou la compréhension de l'évolution de certaines pratiques alimentaires. Les trois exemples présentés cidessous donnent de telles analyses croisées.

\subsection{Lexicographie et restes archéobotaniques : la «plante à huile » est-elle du lin ou du sésame ?}

L'huile est fréquemment mentionnée dans les textes cunéiformes car, outre son utilisation dans l'alimentation, elle sert aux soins du corps, à la parfumerie et à l'éclairage. La principale plante oléagineuse cultivée en Mésopotamie est désignée dans les textes par le sumérien ŠE.GIŠ.İ, «grain à huile végétale », et l'akkadien šamaššammū (̌́aman šammū, littéralement « la plante à huile ») ${ }^{57}$. Il ne peut s'agir de l'olivier, principalement cultivé dans les régions de climat méditerranéen et dont l'huile est importée en Mésopotamie dès la fin du III $^{\mathrm{e}}$ millénaire depuis la côte syro-palestinienne. En raison de la ressemblance entre le mot akkadien

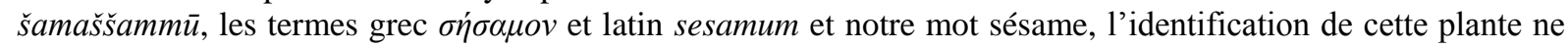
semblait pas poser problème. Mais en 1966, un archéobotaniste, H. Haelbeck, remet cette identification en question car, à cette date, aucune graine de sésame n'a été identifiée, pour les niveaux antérieurs au $\mathrm{I}^{\mathrm{er}}$ millénaire av. J.-C., dans les restes végétaux exhumés sur les chantiers archéologiques du Proche-Orient ancien. Il propose d'y voir plutôt le nom du lin, l'une des toutes premières plantes domestiquées, dont les graines sont abondantes au Proche-Orient dès le $\mathrm{VI}^{\mathrm{e}}$ millénaire ${ }^{58}$. Selon $\mathrm{H}$. Haelbeck, il y aurait eu une redénomination au $\mathrm{I}^{\mathrm{er}}$ millénaire, période où les graines de sésame sont attestées et où les descriptions textuelles désignent clairement le sésame. Le débat sur l'identification de la « plante à huile » n'est pas encore complètement clôt aujourd'hui.

L'iconographie n'est d'aucune aide pour cette question, car il n'existe pas de représentation de lin ou de sésame pour cette région. En revanche, les sources sumériennes et akkadiennes du début du II $^{\mathrm{e}}$ millénaire, en particulier l'Almanach du fermier, documentent le cycle de la plante ŠE.GIŠ.İ qui s'applique parfaitement au sésame, semé au printemps et récolté à la fin de l'été, mais pas du tout au lin dont la croissance a lieu l'hiver" Ce débat donne naissance, en 1985, au deuxième volume du Bulletin on Sumerian Agriculture où contribuent archéo- et ethno-botanistes, ainsi qu'épigraphistes, selon lesquels l'introduction du sésame au Proche-Orient daterait du milieu du III $^{\mathrm{e}}$ millénaire.

L'absence de graines de sésame dans les sites mésopotamiens n'est pas un argument suffisant, étant donnée la fragilité des graines de sésame et leur petite taille comparée à celle des graines de lin, et surtout le manque de finesse des fouilles proche-orientales jusqu'à une époque relativement récente. De fait, l'argumentation a silencio avancée par H. Haelbeck ne tient plus guère aujourd'hui alors que des graines de

\footnotetext{
53 Tengberg 2009.

54 Joannès 2009a.

${ }^{55}$ Milano s. p.

${ }^{56}$ Zarnkow et al. 2006.

${ }^{57}$ Cette section repose sur l'étude de Reculeau 2009. Voir également Powell 1991.

${ }^{58}$ L'usage des fibres végétales de lin remonte aux débuts de l'agriculture vers 9000 avant notre ère. Le lin est attesté par les textes consacrés à la production textile sous les termes sumérien GU et akkadien gî et kitû.

${ }^{59}$ Civil 1994.
} 
in B. Lion (éd.), L'histoire de l'alimentation dans l'Antiquité : Bilan historiographique, Dialogues d'Histoire Ancienne Supplément 7, Besançon, 2012, p. 17-45.

sésame ont été découvertes en Mésopotamie, à Abu-Șalabih, pour la première moitié du $\mathrm{III}^{\mathrm{e}}$ millénaire avant J.C. ${ }^{60}$. Malgré ces nouvelles découvertes, certains auteurs persistent à identifier la plante à huile au lin.

\subsection{Une illustration archéologique du système des rations alimentaires ?}

Dans les grandes institutions mésopotamiennes, la production quotidienne est normalisée et comptabilisée sur l'année. Les travailleurs (GURUŠ, GÉME) sont rétribués en nature, par des rations d'orge (S̆E.BA), d'huile et de laine (2 $\mathrm{kg}$ par an pour un adulte), organisées généralement tous les mois ${ }^{61}$. L'huile est versée en petites quantité, 5 litres par an. Le volume de la ration d'orge est fixé selon le sexe et l'âge. Selon l'article fondateur d'I. J. Gelb, publié en 1965, à l'époque d'Ur III (fin du III ${ }^{\mathrm{e}}$ millénaire), un homme reçoit en moyenne 21 . d'orge par jour (le litre sumérien représente environ 0,8 de notre litre), une femme 1 l. d'orge seulement et un enfant ou un vieillard entre 0,3 et 0,61 . par jour. Ces données semblent juste correspondre aux besoins caloriques nécessaires quotidiennement pour chaque classe d'âge; mais beaucoup de travailleurs assurent des tâches pénibles. Quelques chercheurs proposent les calculs suivants : 1 litre d'orge battu (ration quotidienne d'une travailleuse) $=2700 \mathrm{Kcal}$; or le besoin énergétique moyen d'une femme aujourd'hui est de 2200 Kcal. En l'absence d'autres aliments, il s'agit là d'un minimum vital. Ces distributions sont individuelles : les enfants entrent très tôt dans les équipes de travailleurs et reçoivent leur propre ration.

Ce système de rétribution a, sans doute, laissé des traces archéologiques. En 1970, H. Nissen propose que les bols à forme tronconique à bord biseauté (bevelled rim bowls), de fabrication grossière et rapide, produits en série, et découverts en très grand nombre sur les sites du $\mathrm{IV}^{\mathrm{e}}$ millénaire dans tout le Proche-Orient ancien, aient été fabriqués dans le cadre de la distribution des rations alimentaires. Leur forme et taille uniformes / standardisées iraient dans ce sens ${ }^{62}$. Ces récipients présenteraient principalement trois types de volumes : 0,9 litre / 0,65 litre / 0,45 litre correspondant à différentes rations ${ }^{63}$. Au $\mathrm{III}^{\mathrm{e}}$ millénaire, ces écuelles grossières sont remplacées par diverses écuelles coniques tournées dites «pots de fleur» et présentant des volumes similaires ; on trouve aussi les «SÌLA bowls», d'une capacité d'un litre.

Plusieurs auteurs objectent à cette interprétation la taille trop petite de ce bol pour contenir une ration à moins d'être rempli en dôme (!), les variations morphologiques qui ne cadrent pas avec une centralisation de la production, l'aspect inadapté pour le transport et les contextes de découverte très variés ${ }^{64}$. On pourrait ajouter que la majorité des textes mentionnent un versement mensuel et non quotidien des rations (sauf à Ebla). D'autres interprétations sont avancées pour ces récipients : fonction votive, vaisselle jetable pour repas communautaires, mesures pour le versement d'impôts, moules à pain (comme dans l'iconographie égyptienne), voire moule pour faire des pains de $\operatorname{sel}^{65}$. Mais l'hypothèse la plus fréquemment retenue fait de ces récipients des mesures pour les rations alimentaires pour les travailleurs ${ }^{66}$.

\subsection{Les suidés, de la domestication au tabou sur la viande de porc dans le Proche-Orient}

Autre type de problématique qui a vu le jour ces dernières années: combiner les données archéologiques, épigraphiques et textuelles pour suivre sur le long terme l'évolution des relations entre l'homme et une espèce animale comestible. Les suidés, ont fait l'objet d'un colloque en 2005 , dont les actes ont été publiés l'année suivante ${ }^{67}$. Élevé au départ de manière générale dans tout le Proche-Orient, le cochon en est ensuite banni par les tabous formulés dans la Bible et dans le Coran. Contrairement aux autres animaux de rente, domestiqués à partir du $\mathrm{X}^{\mathrm{e}}$ millénaire av. J.-C. au Proche-Orient et qui procurent à l'homme viande, lait, peau, laine et poils, ainsi qu'une force de travail concernant le gros bétail, le cochon n'est élevé que pour sa viande et sa graisse.

Vers la fin du IV e millénaire, des sangliers apparaissent en contexte cynégétique sur les scènes miniatures gravées sur les sceaux-cylindres, et l'existence de suidés est confirmée par les restes fauniques découverts en Mésopotamie et en Syrie. Les listes lexicales hésitent sur le classement de l'animal, entre sauvage et domestique : même si les troupeaux de porcs sont attestés par les textes dès le $\mathrm{IV}^{\mathrm{e}}$ millénaire, dans la seconde moitié du III ${ }^{\mathrm{e}}$ millénaire, elles rangent le cochon avec les animaux sauvages. Aux $\mathrm{III}^{\mathrm{e}}$ et $\mathrm{II}^{\mathrm{e}}$ millénaires av. J.-C., à côté des troupeaux de cochons dépendant des palais ou des grands domaines, les particuliers élèvent quelques

\footnotetext{
${ }^{60}$ Charles 1993.

${ }^{61}$ À titre d'exemple pour la fin du IIIe millénaire, cf. Beaugeard 2009 et pour la seconde moitié du Ier millénaire, Tolini 2009c.

${ }^{62}$ Nissen 1970. Voir la synthèse proposée par Faivre 2009c.

${ }^{63}$ Faivre 2009c.

${ }^{64}$ Beale 1978.

${ }^{65}$ Beale 1978 ; Forest 1987; Nicholas 1987 ; Millard 1988 ; Buccellati 1990.

${ }^{66}$ Frangipane 1989, pour Arslantepe.

${ }^{67}$ Lion \& Michel 2006. Cette section est un résumé des différentes contributions publiées dans cet ouvrage.
} 
in B. Lion (éd.), L'histoire de l'alimentation dans l'Antiquité : Bilan historiographique, Dialogues d'Histoire Ancienne Supplément 7, Besançon, 2012, p. 17-45.

bêtes, nourries avec des déchets. La viande de porc est consommée, sa graisse est utilisée en cuisine et quelques parties de l'animal servent en pharmacopée. Si les proverbes mettent en valeur l'image de prospérité donnée par la fertilité de la truie, la littérature divinatoire de la fin du $\mathrm{II}^{\mathrm{e}}$ millénaire associe souvent à l'animal des présages négatifs. Au I ${ }^{\text {er }}$ millénaire, l'animal est toujours élevé en Assyrie et en Babylonie, dans les domaines ruraux et sans doute aussi dans les villes. Mais il apparaît avec une connotation péjorative dans les textes littéraires provenant des milieux cléricaux : il est sale, stupide et symbole d'impureté rituelle.

$\mathrm{Au}$ Levant sud, le cochon est bien attesté jusqu'au milieu du $\mathrm{II}^{\mathrm{e}}$ millénaire, mais disparaît à la fin du $\mathrm{II}^{\mathrm{e}}$ millénaire. Au début du $\mathrm{I}^{\mathrm{er}}$ millénaire, il reparaît sur quelques sites, mais cela ne suffit pas pour en faire un marqueur ethnographique comme certains furent tentés de le faire ${ }^{68}$. La Bible classe l'animal parmi les animaux qu'il est interdit de manger car « il a le sabot fendu mais ne rumine pas » ${ }^{69}$, interdit que reprend ensuite $1^{\prime}$ Islam $^{70}$. On constate, au $\mathrm{I}^{\mathrm{er}}$ millénaire, un déclin général de l'élevage des suidés dans l'ensemble du Proche-Orient.

Plusieurs explications semblent expliquer la présence ou l'absence de cochons. La sécheresse du climat ou le mode de vie nomade de certains groupes sont défavorable à son élevage; de plus, le sud de la Mésopotamie se prête davantage à l'élevage spécialisé des petits ruminants, moutons et chèvres, qu'à un élevage diversifié incluant le porc. Le choix de privilégier l'élevage d'un animal ou un autre tient aussi à des logiques socio-économiques et politiques. Les choix humains sont des facteurs déterminants. Pour les anthropologues, en dehors des interprétations économiques, politiques ou écologiques, l'interdiction de consommation de viande de porc, ou au contraire l'incitation à en consommer, dans certaines religions, résulterait d'une nécessité de se distinguer d'autres groupes, en instaurant ou en refusant le tabou ${ }^{71}$.

Les travaux sur l'alimentation au Proche-Orient ancien, extrêmement nombreux et variés, ont vu un renouvellement de leurs problématiques depuis quelques décennies : nouvelles sources, nouvelles thématiques, nouvelles techniques d'analyses. Que reste-t-il aujourd'hui de l'alimentation des anciens Mésopotamiens, audelà des expérimentations sur la bière ? En publiant les trois seules tablettes de recettes babyloniennes connues à

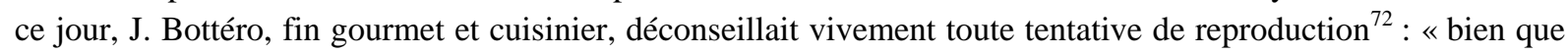
je les tienne (les ancien Mésopotamiens), pièces en main, pour les premiers inventeurs et pratiquants d'une gastronomie et d'une grande cuisine, je ne conseillerai à personne d'incorporer à la nôtre leur tradition culinaire, telle quelle ». Malgré ces mises en garde fondées sur une évolution importante des goûts en quatre mille ans, certains s'y sont essayé avec plus ou moins de succès. Depuis plus d'une décennie, à Brown University, plus d'une centaine de dîners babyloniens sont servis lors d'une réception annuelle. J. Bottéro n'avait pas besoin de tester ces recettes pour imaginer que leur goût était proche « de cette cuisine 'arabo-turque', 'libanaise', ou 'proche-orientale' (qu'on l'appelle comme on voudra), à notre portée, et dont il est assez vraisemblable, sur le plan de l'Histoire, qu'elle constitue, en somme, le prolongement, la présentation contemporaine, et la seule accessible, de la vieille technique mésopotamienne, égarée, du Manger et du Boire, de la Cuisine et de la Table $»^{73}$.

\author{
Abréviation \\ BSA Bulletin on Sumerian Agriculture, J. N. Postgate \& M. Powell (éd.), Cambridge: \\ Vol. 1: (Céréales), 1984. \\ Vol. 2: (Céréales et légumineuses), 1985. \\ Vol. 3: (Fruits et legumes), 1987. \\ Vol. 4: Irrigation and Cultivation in Mesopotamia, Part 1, 1988. \\ Vol. 5: Irrigation and Cultivation in Mesopotamia, Part 2, 1990. \\ Vol. 6: Trees and Timber in Mesopotamia, 1992. \\ Vol. 7: Domestic Animals of Mesopotamia, Part 1, 1993. \\ Vol. 8: Domestic Animals of Mesopotamia, Part 2, 1995.
}

\footnotetext{
${ }^{68}$ Finkelstein \& Silberman 2002.

${ }^{69}$ Lv 11, 7, cf. Douglas 2001.

${ }^{70}$ « Il ne prohibe pour vous que les chairs mortes, le sang, la viande de porc ou dédiée à un autre que Dieu. Qui en consomme toutefois par nécessité, non par insolence non plus que par transgression, sur lui point de péché », Coran 2, 173 ; voir aussi les versets 5,3;6, 145 et 16,115. Les légistes ont étendu l'interdiction à tout ce qui provient du porc : la chair, la graisse, les os, la peau et les poils. Il ne faut ni posséder un porc, ni en faire transaction. Cf. Benkheira 2000.

${ }^{71}$ Fabre-Vassas 1994.

${ }^{72}$ Bottéro 1982, p. 82.

${ }^{73}$ Bottéro 2002, 19.
} 
in B. Lion (éd.), L'histoire de l'alimentation dans l'Antiquité : Bilan historiographique, Dialogues d'Histoire Ancienne Supplément 7, Besançon, 2012, p. 17-45.

\section{Bibliographie}

Amigues, S. (2003) : « Pour la table du Grand Roi », Journal des Savants, janvier-juin, 3-59.

Bachelot, L. (2009a) : « À boire et à manger en images », in Michel 2009a, 439-446 (en ligne à l'adresse http://www.mae.uparis10.fr/cahiers/pdf/C9/C9 T9 Bachelot1.pdf).

Bachelot, L. (2009b) : « Le matériel funéraire lié à l'alimentation (des morts ?) », in Michel 2009a, 475-483 (article en ligne à l'adresse http://www.mae.u-paris10.fr/cahiers/pdf/C9/C9 T9 Bachelot2.pdf).

Barnett, D. (1985) : “Assurbanipal's Feast”, Eretz-Isael 18, 1-6.

Beale, T.W. (1978): "Bevelled Rim Bowls and Their Implications for Change and Economic Organization in the Later Fourth Millennium BC", Journal of Near Eastern Studies 37, 289-313.

Beaugeard, C. (2009) : "Quelques données sur les systèmes de rationnement à la fin du IIIe millénaire », in Michel 2009a, 321-325 (en ligne à l'adresse http://www.mae.u-paris10.fr/cahiers/pdf/C9/C9 T9 Beaugeard.pdf).

Benkheira, M. H. (2000) : Islam et interdits alimentaires, juguler l'animalité, Paris.

Birot, M. (1960): Textes administratifs de la salle 5 du palais (1), Paris (Archives royales de Mari 9).

Birot, M. (1964) : Textes administratifs de la salle 5 du palais (2), Paris (Archives royales de Mari 12).

Bordreuil, P., F. Briquel-Chatonnet et C. Michel, éd. (2008) : Les débuts de l'histoire. Le Proche-Orient de l'invention de l'écriture à la naissance du monothéisme, Paris.

Bottéro, J. (1957) : Textes économiques et administratifs, Paris (Archives royales de Mari 7).

Bottéro, J. (1982) : « La plus vieille cuisine du monde », L'Histoire 49, 72-82.

Bottéro, J. (1992a) : L'épopée de Gilgamesh. Le grand homme qui ne voulait pas mourir, Paris.

Bottéro, J. (1992b) : "Konservierung von Lebensmitteln", Reallexicon der Assyriologie 6, 191-197.

Bottéro, J. (1995): Textes culinaires mésopotamiens/Mesopotamian Culinary Texts, Winona Lake (Mesopotamian Civilizations 6).

Bottéro, J. (2002) : La plus vieille cuisine du monde, Paris.

Bouchaud, C. (2009) : «Les pratiques alimentaires en Arabie antique. Étude archéobotanique de Madâ'in Sâlih », in Michel 2009a, 247-256 (en ligne à l'adresse http://www.mae.u-paris10.fr/cahiers/pdf/C9/C9 T9 Bouchaud.pdf).

Bray, T. L., éd. (2003) : The Archaeology and Politics of Food and Feasting in Early States and Empires, New York, Boston, Dordrecht, Londres, Moscou.

Breniquet, C. (2009a) : " Boire de la bière en Mésopotamie... », in Faivre, Lion \& Michel 2009, 183-196.

Breniquet, C. (2009b) : "Buvait-on de la bière au chalumeau en Mésopotamie à l'époque protodynastique ? ", in Michel 2009a, 359-365 (article en ligne à l'adresse http://www.mae.u-paris10.fr/cahiers/pdf/C9/C9 T9 Breniquet.pdf).

Briant, P. (1989) : « Table du roi, Tribut et redistribution chez les Achéménides », in Briant \& Herrenschmidt 1989, 35-44.

Briant, P. et C. Herrenschmidt, éd. (1989) : Le tribut dans l'empire perse. Actes de la table ronde de Paris, 12-13 décembre 1986, Paris.

Brunke, H. (2011): Essen in Sumer. Metrologie, Herstellung und Terminologie nach Zeugnis der UrIII-zeitlichen Wirtschaftsukunden, Munich (Gescgucgtswussebscgafteb 26).

Buccellati, G. (1990) : "Salt at the Dawn of History : The Case of the Bevelled-Rim Bowls", in Matthiae et al., éd. 1990, $17-$ 37.

Burke, M. L. (1968) : Textes administratifs de la salle 111 du palais, Paris (Archives royales de Mari 11).

Calmeyer, P. (1987/1990) : "Mahlzeit. C. Archäologisch", Reallexikon der Assyriologie 7, 270-271.

Cavigneaux, A. (1983) : « Lexikalische listen A. », Reallexikon der Assyriologie 6, 609-641.

Chambon, G. (2009) : Les Archives du vin à Mari, Paris (Florilegium Marianum 11).

Charlier, P. (1987) : «Les glacières à Mari », Akkadica 54, 1-10.

Charles, M. (1993) : "Botanical Remains", in Green 1993, 203-207.

Charpin, D. (1988) : «Les représentants de Mari à Babylone (I) », in : Charpin, Joannès, Lackenbacher \& Lafont B 1988, 139-205.

Charpin, D., F. Joannès, S. Lackenbacher et B. Lafont B. (1988) : Archives épistolaires de Mari I/2, Paris (Archives Royales de Mari 26/2).

Charpin, D. et J.-M. Durand, éd. (1994) : Recueil d'études à la mémoire de Maurice Birot, Paris (Florilegium Marianum 2).

Charpin, D. et J.-M. Durand, éd. (1997) : Recueil d'études à la mémoire de Marie-Thérèse Barrelet, Paris (Florilegium Marianum 3), Paris.

Civil, M. (1994) : The Farmer's Instructions, A Sumerian Agricultural Manual, Barcelone.

Collon, D. (1992) : "Banquets in the Art of the Ancient Near East", in Gyselen 1992, 23-30.

Dentzer, J.-M. (1982) : Le motif du banquet couché dans le Proche-Orient et le monde grec du VII au IVe siècle avant J.-C., Paris.

De Odorico, M. (1995) : Numbers and Quantifications in the Assyrian Royal Inscritpions, Helsinki (State Archives of Assyria Studies 3).

Dercksen, J. G. (2008) : "Subsistence, surplus and the market for grain and meat at ancient Kanesh", Altorientalische Forschungen 35, 86-102.

Dietler, M. et B. Hayden, éd. (2001) : Feasts. Archaeological and Ethnographical Perspectives on Food, Politics, and Power, Washington et Londres.

Dolce, R. et C. Zaccagnini, éd. (1989): Il pane del re. Accumpulo e distribuzione dei cereali nell'Oriente antico, Bologne.

Douglas, M. (2001) : De la souillure, Paris (première édition 1966).

Durand, J.-M. (1983) : Textes administratifs des salles 134 et 160 du palais de Mari, Paris (Archives royales de Mari 21).

Durand, J.-M. (1997) : Documents épistolaires du palais de Mari, Tome 1, Paris (Littérature ancienne du Proche-Orient 16).

Durand, J.-M. et D. Charpin, éd. (2001) : Mari, Ebla et les Hourrites, dix ans de travaux, Paris (Amurru 2). 
in B. Lion (éd.), L'histoire de l'alimentation dans l'Antiquité : Bilan historiographique, Dialogues d'Histoire Ancienne Supplément 7, Besançon, 2012, p. 17-45.

Durand, J.-M. et A. Jacquet, éd. (s. p.) : La fête au Palais : banquets, parures et musique en Orient, Actes du colloque organisé par l'Institut du Proche-Orient Ancien du Collège de France, la Société Asiatique et le CNRS (UMR 7192), les 29 et 30 mai 2007, Paris.

Durand, J.-M. et J.-R. Kupper, éd. (1985) : Miscellanea Babylonica. Mélanges offerts à Maurice Birot, Paris.

Englund, R. K. (1991) : "Hard work - Where will it get you? Labor Management in Ur III Mesopotamia", Journal of Near Eastern Studies 50, 255-280.

Fabre-Vassas, C. (1994) : La bête singulière, les Juifs, les chrétiens et le cochon, Paris.

Faivre, X. (2009a) : « Pots et plats », in Faivre, Lion \& Michel 2009, 157-182.

Faivre, X. (2009b) : «Récipients, ustensiles et alimentation : fonctions et usages multiples... mais lesquels ? », in Michel 2009a, 277-294 (en ligne à l'adresse http://www.mae.u-paris10.fr/cahiers/pdf/C9/C9 T9 Faivre1.pdf).

Faivre, X. (2009c) : « Rations et notion de capacité standard dans la céramique du Proche-Orient », in Michel 2009a, 305-319 (en ligne à l'adresse http://www.mae.u-paris10.fr/cahiers/pdf/C9/C9_T9_Faivre2.pdf).

Faivre, X. (2009d) : "Vases à bière : de la production à la consommation », in Michel 2009a, 367-383 (article en ligne à l'adresse http://www.mae.u-paris10.fr/cahiers/pdf/C9/C9 T9 Faivre3.pdf).

Faivre, X., B. Lion, et C. Michel, éd. (2009), Et il y eut un esprit dans l'Homme. Jean Bottéro et la Mésopotamie, Paris (Travaux de la Maison René-Ginouvès 6), particulièrement chapitre intitulé « La plus vieille cuisine du monde », $157-254$

Finet, A. (1992) : « Le Banquet de Kalah offert par le roi d'Assyrie Ašurnazirpal II (883-859) », in Gyselen 1992, 31-44.

Finkelstein, I. et N. A. Silberman (2002) : La Bible dévoilée. Les nouvelles révélations de l'archéologie, Paris.

Forest, J.-D. (1987) : «Les Bevelled Rim Bowls. Nouvelle tentative d'interprétation », Akkadica_53, 1-24.

Frangipane, M. (1989) : « Produzione di vasellame in serie e distribuzione di razioni alimentari nelle società proturbane del periodo tardo Uruk-Jemdet Nasr", in Dolce \& Zaccagnini 1989, 49-63.

Gelb, I. J. (1965) : “The Ancient Mesopotamian Ration System”, Journal of Near Eastern Studies 24, 230-243.

Glassner, J.-J. (1987/1990) : "Mahlzeit. A. In Mesopotamien”, Reallexikon der Assyriologie 7, 259-267.

Gouin, P. (1993) : « Bovins et laitages en Mésopotamie méridionale au $3^{\mathrm{e}}$ millénaire », Iraq 55, 135-145.

Green, A., éd. (1993) : Abu Salabikh Excavations Volume 4. The 6G Ash-Tip and its contents : cultic and administrative discard from the temple?, Londres (British School of Archaeology in Iraq).

Guichard, M. (1997) : «Le sel à Mari (III) : les lieux du sel », in Charpin \& Durand 1997, 167-173.

Guichard, M. (2005) : La vaisselle de luxe des rois de Mari, Paris (Archives Royales de Mari 31).

Gyselen, R., éd. (1992) : Banquets d'Orient, Bures-sur-Yvette (Res Orientales 4).

Haelbeck, H. (1966) : "The plant remains from Nimrud”, in Mallowan 1966, 613-620.

Hartman, L. F. et A. F. Oppenheim, éd. (1950): On Beer and Brewing Techniques in Ancient Mesopotamia, Baltimore (Supp a. JAOS 10).

Hoffner, H. A. Jr. (1974) : Alimenta Hethaeorum. Food Production in Hitite Asia Minor, New Haven (American Oriental Series 55).

Hugoniot, C., C. Grandjean et B. Lion, éd. (s. p.) : Le banquet du monarque dans le monde antique. Actes du colloque de Tours, 25-27 mars 2010, Tours (Presses Universitaires François-Rabelais).

Joannès, F. (1996) : « La fonction sociale du banquet en Mésopotamie », in Flandrin \& Montanari 1996, 47-60.

Flandrin, J.-L. et M. Montanari, éd. (1996) : Histoire de l'alimentation, Paris.

Joannès, F. (1994) : «L'eau et la glace », in Charpin \& Durand 1994, 137-150.

Joannès, F., dir., assisté de C. Michel (2001) : Dictionnaire de la civilisation mésopotamienne, Paris.

Joannès, F. (2008) : «L'alimentation des élites mésopotamiennes : nourriture du roi, nourriture des dieux », in Leclant, Vauchez \& Sartre 2008, 23-38.

Joannès, F. (2009a) : « La production des dattes d'après la documentation textuelle néo-babylonienne, in Michel 2009a, 243 246 (en ligne à l'adresse http://www.mae.u-paris10.fr/cahiers/pdf/C9/C9 T9 Joannes1.pdf).

Joannès, (2009b) : « La vaisselle d'apparat dans la documentation mésopotamienne du I ${ }^{\text {er }}$ millénaire av. J.-C. », in Michel 2009a, 295-302 (en ligne à l'adresse http://www.mae.u-paris10.fr/cahiers/pdf/C9/C9 T9 Joannes2.pdf).

Joannès, F. (2009d) : « La table des dieux babyloniens », in Michel 2009a, 453-461 (article en ligne à l'adresse http://www.mae.u-paris10.fr/cahiers/pdf/C9/C9 T9 Joannes4.pdf).

Koslova, N. V. (2006) : "Barley Rations in Umma during the Third Dynasty of Ur", Babel und Bibel 3, 41-58.

Lafont, B. (1985) : Le șâbum du roi de Mari au temps de Yasmah-Addu, dans Durand \& Kupper 1985, 161-179.

Lafont, B. (2001): «Relations internationales, alliances et diplomatie au temps des royaumes amorrites - Essai de synthèse ", in Durand \& Charpin 2001, 213-328.

Lafont, B. (2009) : «La consommation de viande à Sumer », in Michel 2009a, 421-430 (article en ligne : http://www.mae.uparis10.fr/cahiers/pdf/C9/C9 T9 Lafont.pdf)

Lacambre, D. (2009) : «Le bureau de la bière de Chagar Bazar (Syrie), in Michel 2009a, 385-391 (article en ligne: http://www.mae.u-paris10.fr/cahiers/pdf/C9/C9 T9 Lacambre.pdf)

Landsberger, B. (1960) : « The Fauna of Ancient Mesopotamia, First part, Tablet XIII », Rome (Materialien zum sumerischen Lexikon 8/1).

Leclant, J., A. Vauchez et M. Sartre, éd. (2008) : Colloque Pratiques et discours alimentaires en Méditerranée de l'Antiquité à la Renaissance, Actes, Paris.

Lion, B. (2009) : « Nourrir les morts, d'après la documentation épigraphique », in Michel 2009a, 465-473 (article en ligne à l'adresse http://www.mae.u-paris10.fr/cahiers/pdf/C9/C9 T9 Lion.pdf).

Lion, B. et C. Michel, (1997) : "Criquets et autres insectes à Mari », Paris (Mari. Annales de Recherches Interdisciplinaires 8), 707-724.

Lion, B. et C. Michel, (2000) : «Poissons et crustacés en Haute-Mésopotamie au début du $\mathrm{II}^{\mathrm{e}}$ millénaire av. J.-C., Topoi, Supplément $n^{\circ} 2,71-116$. 
in B. Lion (éd.), L'histoire de l'alimentation dans l'Antiquité : Bilan historiographique, Dialogues d'Histoire Ancienne Supplément 7, Besançon, 2012, p. 17-45.

Lion, B. et C. Michel, éd. (2003), Banquets et fêtes au Proche-Orient ancien, Dossiers d'Archéologie 280, février.

Lion, B. et C. Michel, éd. (2006) : De la domestication au tabou : le cas des suidés au Proche-Orient ancien, Paris (Travaux de la Maison René-Ginouvès 1).

Maekawa, K. (1989) : "Rations, Wages and Economic Trends in The Ur III Period”, Altorientalische Forschungen 16, 42-50.

Maekawa, K. (1998) : "Ur III Girsu Records of Labor Forces in the British Museum (1)”, Acta Sumerologica Japan 20, 63110.

Mallowan, M. E. L., éd. (1966) : Nimrud and its remains, Londres.

Margueron, J.-C. (2004) : Mari. Métropole de l'Euphrate au III' et au début II millénaire av. J.-C., Paris.

Marro, C. et C. Michel (s. p.) : «Le sel dans les sociétés anciennes du Proche-Orient et du Caucase : exploitations et usages d'après les sources archéologiques et épigraphiques ", Cahiers des thèmes transversaux d'ArScAn XI (2009-2010), Nanterre.

Matthiae et al. (éd.) 1990 : Resurrecting the Past : A Joint Tribute to Adnan Bounni, Istanbul.

Matthiae, P. (1985) : I tresori di Ebla, Rome-Bari.

Matthiae, P. (1998) : Ninive, Milan.

Michalowski, P. (1994) : "The Drinking Gods : Alcohol in Mesopotamian Ritual”, in Milano 1994, 27-44.

Michalowski, P., éd. (2008) : On the Third Dynasty of Ur. Studies in Honor of Marcel Sigrist, Boston.

Michel, C. (1997) : « À table avec les marchands paléo-assyriens », in Waetzoeldt \& Hauptmann 1997, 95-113.

Michel, C. (2006) : «Les suidés dans la documentation de Kaniš au début du II ${ }^{\mathrm{e}}$ millénaire avant J.-C. », in Lion \& Michel, $169-180$

Michel, C., éd. (2009a) : L'alimentation dans l'Orient ancien, de la production à la consommation, Thème IX, Cahiers des thèmes transversaux d'ArScAn IX (2007-2008), Nanterre (en ligne à l'adresse http://www.mae.uparis 10.fr/arscan/L-alimentation-dans-1-Orient, 888.html).

Michel, C. (2009b) : «Le transport des denrées alimentaires dans la documentation écrite du début du $\mathrm{II}^{\mathrm{e}}$ millénaire », in Michel 2009a, 265-273 (article en ligne : http://www.mae.u-paris10.fr/cahiers/pdf/C9/C9 T9 Michel2.pdf)

Michel, C. (2009c) : «'Dis-moi ce que tu bois...' Boissons et buveurs en haute Mésopotamie et Anatolie au début du II ${ }^{\mathrm{e}}$ millénaire av. J.-C. », in Faivre, Lion \& Michel 2009, 197-220.

Michel, C. (2009d) : «Les boissons en Mésopotamie du nord et Anatolie dans la première moitié du IIe millénaire av. J.-C. », in Michel 2009a, 351-358 (article en ligne à l'adresse http://www.mae.uparis10.fr/cahiers/pdf/C9/C9 T9 Michel3.pdf).

Milano, L. éd. (1994) : Drinking in Ancient Societies; History and Culture of Drinks in the Ancient near East: Papers of a Symposium Held in Rome, May 17-19, 1990, Padoue (History of the Ancient Near East / Studies 6).

Milano, L. éd. (s. p.) : Methods and Perspectives applied to the Study of Food Practices in the Ancient Near East, Padoue (History of the Ancient Near East / Studies).

Millard, A.M. (1988) : "The Bevelled-Rim Bowls : their Purpose and significance”, Iraq 50, 49-57.

Nicholas, I.M. (1987) : “The Function of Bevelled-Rim Bowls : A Case Study at the TUV Mound, Tal-e-Malyan”, Paléorient $13 / 2,61-72$.

Nissen, H.J. (1970) : “Grabung in Quadraten K/L XII in Uruk-Warka”, Baghdader Mitteilungen 5, 101-191.

Parpola, S. (2004) : "The Leftovers of God and King. On the Distribution of Meat at the Assyrian and Achaemenid Court", in Grottanelli \& Milano 2004, 281-312.

Parrot, A. (1959) : Le Palais. Documents et Monuments, Paris (Mission archéologique de Mari II).

Patrier, J. (2009a) : « Conservation et stockage des denrées alimentaires au Proche-Orient ancien au IIe millénaire av. J.-C. : une approche méthodologique », in Michel 2009a, 335-347 (article en ligne à l'adresse http://www.mae.uparis10.fr/cahiers/pdf/C9/C9 T9 Patrier1.pdf).

Patrier, J. (2009b) : «L'alimentation des morts en Anatolie au IIe millénaire av. J.-C. : une étude préliminaire », in Michel 2009a, 485-493 (article en ligne à l'adresse http://www.mae.u-paris10.fr/cahiers/pdf/C9/C9_T9_Patrier2.pdf).

Patrier, J. (2011) : Conservation et stockage des denrées alimentaires en Anatolie centrale au IIe millénaire av. J.-C., Strasbourg (thèse inédite).

Pinnock, F. (1994) : "Considerations on the 'Banquet Theme' in the Figurative Art of Mesopotamia and Syria", in Milano 1994, 15-26 et pl. I-IX.

Pollock, S. (2003) : "Feasts, Funerals, and Fast Food in Early Mesopotamian States", in Bray 2003, 17-38.

Powell, M. A., éd., (1987) : Labor in the Ancient Near East, New Haven (American Oriental Society 68).

Powell, M. A. (1991) : "Epistemology and Sumerian Agriculture: The Strange Case of Sesame and Linseed", Aula Orientalis 9, 155-164.

Reculeau, H. (2009) : « Le point sur la « plante à huile » : réflexions sur la culture du sésame en Syrie-Mésopotamie à l'âge du Bronze », Journal des Médecines cunéiformes 13, 13-37.

Reiner, E. et M. Civil (1974): The Series HAR-ra = hubullu. Tablets XX-XXIV, Rome (Materials for the Sumerian Lexicon 11).

Röllig, W. (1970) : Das Bier im Alten Mesopotamien, Berlin.

Sasson, J. M. (2004) : "The King's Table: Food and Fealty in Old Babylonian Mari”, in Grottanelli \& Milano 2004, 179-215.

Schmandt-Besserat, D. (2001) : "Feasting in the Ancient Near East", in Dietler \& Hayden 2001, 391-403.

Seeher, J. (2000) : "Getreidelagerung in Unterirdischen Großspeichern: Zur Methode und ihrer Anwendung im 2. Jahrtausend v. Chr. Am Beispiel der Befund in Hattuša", Studi micenei ed egeo-anatolici 42, 261-301.

Selz, G. (1983): Die Bankettszene. Entwicklung eines "überzeitlichen" Bildmotivs in Mesopotamien, von der Frühdynastischen bis zur Akkad-Zeit, Wiesbaden (Freiburger altorientalische Studien 11).

Sigrist, M. (1992): Drehem, Bethesda (CDL Press).

Steinkeller, P. (1987) : "The Foresters of Umma: Toward a Definition of Ur III Labor", in Powell 1987, 73-115.

Steinkeller, P. (1996) : "The Organization of Crafts in Third Millennium Babylonia : The Case of Potters", Altorientalische Forschungen 23, 232-253. 
in B. Lion (éd.), L'histoire de l'alimentation dans l'Antiquité : Bilan historiographique, Dialogues d'Histoire Ancienne Supplément 7, Besançon, 2012, p. 17-45.

Steinkeller, P. (2008): “Joys of Cooking in Ur III Babylonia”, in Michalowski 2008, 185-192.

Stol, M. (1971) : «Zur altmesopotamischen Bierbereitung », Bibliotheca Orientalis 28, 167-171.

Tengberg, M. (2009) : « Cultures et utilisations du palmier dattier au Moyen-Orient ancien. Données archéobotaniques », in Michel 2009a, 237-242 (en ligne à l'adresse http://www.mae.u-paris10.fr/cahiers/pdf/C9/C9 T9 Tengberg.pdf).

Tolini, G. (2009a) : « Les repas du Grand Roi en Babylonie : Cambyse et le palais d'Abanu », in Faivre, Lion \& Michel 2009, 237-254.

Tolini, G. (2009b) : «La Table du Grand Roi », in Michel 2009a, 447-451 (en ligne à l'adresse http://www.mae.uparis10.fr/cahiers/pdf/C9/C9 T9 Tolini2.pdf).

Tolini, G. (2009c) : « Les rations alimentaires des oblats des temples de la Babylonie achéménide », in Michel 2009a, 327332 (en ligne à l'adresse http://www.mae.u-paris10.fr/cahiers/pdf/C9/C9 T9_Tolini1.pdf).

Van der Stede, V. (2010): Les pratiques de stockage au Proche-Orient ancien du Natoufien à la première moitié du troisième millénaire avant notre ère, Louvain (Orientalia Lovaniensia Analecta 190).

Vila, E. (1998) : L'exploitation des animaux en Mésopotamie au IV ème et IIIème millénaires avant J.- C., Sophia Antipolis (Monographies du C. R. A. 21).

Vila, E. (s. p.) : «L'approche de la boucherie en archéozoologie : données préliminaires de la découpe du mouton à Mishrifeh/Qatna (Syrie, Bronze récent) » Cahiers des thèmes transversaux d'ArScAn XI (2009-2010), Nanterre.

Waetzoldt, H. (1987) : "Compensation of Craft Workers and Officials in the Ur III Period”, in Powell, M. 1987, 117-141.

Waetzoldt, H. et H. Hauptmann, éd. (1997) : Assyrien im Wandel der Zeiten, Heidelberg.

Zarnkow, M. et al. (2006) : «Interdisziplinäre Untersuchungen zum altorientalischen Bierbrauen in der Siedlung von Tall Bazi/Nordsyrien vor rund 3200 Jahren », Technikgeschichte 73, 3-25. 